\title{
JUST-IN-TIME RAIL SHUTTLE SERVICE FEASIBILITY STUDY FOR THE PORT OF VALENCIA
}

\author{
LORENA SÁEZ-CARRAMOLINO, ALEX SÁNCHEZ-PÉREZ, CARLES PÉREZ-CERVERA \\ \& SALVADOR FURIÓ-PRUÑONOSA \\ Fundación Valenciaport, Spain
}

\begin{abstract}
The growth in container transport volumes, the increase in the size of ships and the concentration of flows through a limited number of port hubs require higher capacity on hinterland connections. Road transport now accounts for most of the connections between ports and hinterland areas in Europe, resulting in port congestion associated with delays, waiting lines and increased permanence of ships and cargo in the port. This translates into additional costs and a negative environmental impact. In most cases, the development of rail transport becomes part of the solution to this problem. The current study contributes to the development of the strategy of the Port of Valencia in order to increase the rail modal share for import/export cargo through the definition and feasibility analysis of an innovative Just-InTime (JIT) Rail Shuttle service for a key port-hinterland corridor in Spain connecting Valencia with Zaragoza. The proposed solution aims to directly unload the containers from the ship and load them onto trains, in order to minimize the movement of containers at the terminal and to operate as an "air bridge" at the airports, so that the shuttle makes round trips within one day and the containers are loaded on the first available JIT rail service. In order to minimize the cost per unit transported the feasibility study includes designing the operational solution(service characteristics, the requirements of the information system and the definition of the business models needed for its implementation.

Keywords: feasibility study, Just-In-Time, port, rail shuttle, rail transport, ship cargo, shipping containers, shuttle service, Spain, Valencia.
\end{abstract}

\section{INTRODUCTION}

The growth in container transport volumes, the increase in the size of ships and the concentration of flows through a limited number of port hubs require higher capacity on hinterland connections. Road transport now accounts for most of the connections between ports and hinterland areas in Europe, resulting in port congestion associated with delays, waiting lines and increased permanence of ships and cargo in the port. This translates into additional costs and a negative environmental impact.

Although ports have developed multiple strategies and policies to improve their hinterland connections, and considering that rail freight transport can better connect these ports to hinterlands, rail freight transport currently has a low share in several EU countries, in which the objectives set up by the European Commission in 2011 (that there should be a modal shift of $30 \%$ of the of the current freight tonnage going above $300 \mathrm{~km}$ from road to rail, by 2030 ; $50 \%$ by 2050 [3]) will not be achieved.

The biggest challenge regarding attracting more shippers to use rail freight is cost reduction, which requires several actions: to improve rail infrastructure; to reduce transit time and increase reliability which has a serious effect on "Just-in-Time" distribution systems [1]; to increase the size of trains and reduce transport costs; to decrease operating costs; to reduce container dwell time enabling cargo owners to save on storage charges that are applied by port terminals; to minimize handling movements per container at port terminals; and to improve communications among actors in the logistic chain allowing a better planning of loading/unloading operations (currently, port terminals receive detailed information about the next transport mode/service only once the container has been unloaded and moved to the 
storage area which results in a waste of time, a lack of efficiency in the supply chain and extra costs [2]).

Currently, port terminals receive information about whether the container will be loaded on a rail service, once the vessel arrives and the container is unloaded and moved to storage areas; which results in a waste of time, a lack of efficiency in the supply chain and an extra cost. In this context, we carried out a study with the objective of analyzing the feasibility of an innovative "Just-In-Time" (JIT) rail shuttle service for the key port-hinterland corridors, in which containers are to be directly unloaded from the vessel and loaded onto trains, minimizing the handling movements. The proposed JIT rail shuttle service operates similarly to an "air bridge" at airports: it travels back and forth at regular intervals over a particular route. Before a container vessel calls to port, the port terminals will know which containers will be directly loaded into the first available JIT rail service, eliminating the use of the container-storage yard.

The solution proposed helps ports to lower their environmental footprint and to move toward cleaner transport modes, supported by disruptive technologies for cargo ports; in order to handle upcoming and future capacity, traffic, efficiency and environmental challenges. Indeed, this innovative JIT rail shuttle service would decrease disturbances to the local population, through a significant reduction in congestion around the port.

This innovative solution fits in closely with a recent way of looking at transport, called the Physical Internet [4]; which will change the way that goods are handled, stored, packaged and transported across the supply chain. It mimics the Digital Internet, as freight in the Physical Internet would travel seamlessly, as data is exchanged through the Internet. Freight transport will become more reliable, efficient and sustainable, if the customer indicates where and when the container has to be delivered; but if at the same time there is more freedom to select the means of transport and the route, so that both transport capacity and transport options can be exploited much more effectively.

\section{METHODOLOGY}

This section describes the methodology used to design an optimal physical and operational solution of JIT rail shuttle service for port-hinterland connections. In particular, we present a case study for connections between the port of Valencia and Zaragoza, for container traffic.

Valenciaport is the leading port in container traffic, in Spain and in the Mediterranean. It is also the 5th European port and the 29th in the world, in this type of traffic; as well as the 4th European port in terms of growth within the last ten years.

The internal railway network of the port of Valencia is directly linked to the Spanish railway network of general interest, which is managed by the Spanish Railway Infrastructure Administrator (ADIF). The railway connections from the port of Valencia ensure access to any manufacturing area on the Iberian Peninsula and in Europe. In this regard, the port of Valencia is connected to the largest logistics platform in Europe, the Zaragoza Logistics Platform (PLAZA), located in Aragon, Spain.

In this context, we see that an efficient connection between Valencia and Aragon is required, in order to increase the hinterland competitiveness of the port of Valencia. As mentioned before, one of the most important factors for attracting more shippers to use rail freight is by way of cost reduction, derived from minimizing handling movements; therefore, the optimal solution will be the one that minimizes the cost per TEU (Twenty-Foot Equivalent Unit) transported. The steps followed to design the optimal JIT rail freight solution are: the definition of inputs and hypothesis; the definition of an optimal train composition model; and a cost analysis including a sensitivity analysis. 


\subsection{Input and hypothesis}

Several inputs are required to design an optimal physical and operational solution of a JIT rail shuttle service. The input variables and hypotheses used in the model are listed, Table 1.

Table 1: Input variables and hypotheses.

\begin{tabular}{|c|c|c|c|c|c|}
\hline \multicolumn{2}{|r|}{ Variable } & Description & Unit & Value & Hypothesis \\
\hline \multirow{2}{*}{ 胥 } & $\begin{array}{l}\text { Door-to-port } \\
\text { railway } \\
\text { distance }\end{array}$ & $\begin{array}{l}\text { Railway distance from the } \\
\text { inland terminal to the } \\
\text { origin/departure port. }\end{array}$ & $\mathrm{Km}$ & 355 & - \\
\hline & $\begin{array}{l}\text { Maximum } \\
\text { train length }\end{array}$ & $\begin{array}{l}\text { Maximum length of a freight } \\
\text { train, including the locomotive. }\end{array}$ & $\mathrm{m}$ & - & 750 \\
\hline \multirow{5}{*}{ 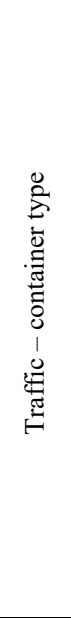 } & $\begin{array}{l}\text { Container } \\
\text { composition }\end{array}$ & $\begin{array}{l}\text { Number of } 20^{\prime}, 40^{\prime} \text { or } 45^{\prime} \\
\text { containers carried on train. }\end{array}$ & $\%$ & - & $\begin{array}{c}20^{\prime}=0-20 \\
40^{\prime}=80 \\
45^{\prime}=0\end{array}$ \\
\hline & Full containers & $\begin{array}{l}\text { Maximum number of full } \\
\text { containers to be transported. }\end{array}$ & $\%$ & - & $0.8-1$ \\
\hline & $\begin{array}{l}\text { Full ITU } \\
\text { weight }\end{array}$ & $\begin{array}{l}\text { An ITU is each }\left(20^{\prime}, 40^{\prime} \text { or } 45^{\prime}\right) \\
\text { container carried by the train. } \\
\text { This variable indicates the } \\
\text { average weight of a full ITU } \\
\text { transported by the } \\
\text { Valencia-Zaragoza rail freight } \\
\text { service. }\end{array}$ & Tons & - & $19-27$ \\
\hline & $\begin{array}{l}\text { ITU tare } \\
\text { weight }\end{array}$ & $\begin{array}{l}\text { Average weight of an empty } \\
\text { ITU transported by the studied } \\
\text { service. }\end{array}$ & Tons & - & $3.46-3.75$ \\
\hline & $\begin{array}{l}\text { Full TEU } \\
\text { weight }\end{array}$ & $\begin{array}{l}\text { Average weight of a full TEU } \\
\text { transported by the service. }\end{array}$ & Tons & - & $11-14$ \\
\hline \multirow{6}{*}{ 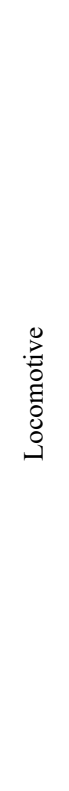 } & $\begin{array}{l}\text { Locomotive } \\
\text { type }\end{array}$ & $\begin{array}{l}\text { Locomotives running on dual } \\
\text { mode (electric-diesel) were } \\
\text { selected: STADLER } \\
\text { Type 1: EURO } 4001 \\
\text { Type 2: EURODUAL }\end{array}$ & - & & $\begin{array}{l}\text { Type } 1 \\
\text { Type } 2\end{array}$ \\
\hline & $\begin{array}{l}\text { Maximum } \\
\text { towable load }\end{array}$ & $\begin{array}{l}\text { Number of cars/wagons that can } \\
\text { be towed by each locomotive, } \\
\text { which depends on the most } \\
\text { unfavorable characteristic ramp } \\
\text { of the section on which the train } \\
\text { runs }(24 \%) \text {. }\end{array}$ & Tons & $\begin{array}{l}\text { EURO } \\
\text { 4001: } 1,020 \\
\text { EURODUA } \\
\text { L: } 1,173\end{array}$ & - \\
\hline & $\begin{array}{l}\text { Fuel } \\
\text { consumption }\end{array}$ & Fuel consumption, diesel mode. & $1 \mathrm{t} / \mathrm{Km}$ & 5.3 & - \\
\hline & Fuel price & $\begin{array}{l}\text { Average price of diesel for } \\
\text { locomotives. }\end{array}$ & $€ / 1 \mathrm{t}$ & - & 0.577 \\
\hline & $\begin{array}{l}\text { Locomotive } \\
\text { acquisition } \\
\text { value }\end{array}$ & $\begin{array}{l}\text { Price that the buyer will pay to } \\
\text { the locomotive manufacturer. }\end{array}$ & Euros & - & $\begin{array}{l}\text { EURO 4001: } \\
\text { 3,700,000 } \\
\text { EURODUAL: } \\
4,200,000\end{array}$ \\
\hline & $\begin{array}{l}\text { Locomotive's } \\
\text { useful life }\end{array}$ & $\begin{array}{l}\text { Estimated number of years the } \\
\text { locomotive is likely to remain in } \\
\text { service. }\end{array}$ & Years & - & 25 \\
\hline
\end{tabular}


Table 1: Continued.

\begin{tabular}{|c|c|c|c|c|c|}
\hline & Variable & Description & Unit & Value & Hypothesis \\
\hline \multirow{5}{*}{ 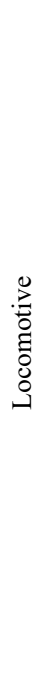 } & $\begin{array}{l}\text { Locomotive's } \\
\text { residual value }\end{array}$ & $\begin{array}{l}\text { Estimated value of the } \\
\text { locomotive at end of its } \\
\text { useful life. }\end{array}$ & $\%$ & & 5 \\
\hline & $\begin{array}{l}\text { Locomotive } \\
\text { annual } \\
\text { depreciation }\end{array}$ & $\begin{array}{l}\text { Locomotive's annual } \\
\text { depreciation }=\text { (Locomotive } \\
\text { acquisition value }- \text { (Locomotive } \\
\text { acquisition value } \times \text { Locomotive } \\
\text { residual value) }) \text { Locomotive } \\
\text { useful life. }\end{array}$ & Euros & - & - \\
\hline & $\begin{array}{l}\text { Locomotive } \\
\text { maintenance } \\
\text { cost per km }\end{array}$ & $\begin{array}{l}\text { Cost covering all locomotive } \\
\text { maintenance tasks, by year. }\end{array}$ & $€ / \mathrm{km}$ & - & 1.3 \\
\hline & $\begin{array}{l}\text { Days of a } \\
\text { replacement } \\
\text { locomotive }\end{array}$ & $\begin{array}{l}\text { Number of days using a } \\
\text { replacement locomotive during } \\
\text { maintenance and repair tasks on } \\
\text { the main locomotive. }\end{array}$ & Days & - & 10 \\
\hline & $\begin{array}{l}\text { Replacement } \\
\text { locomotive } \\
\text { daily cost }\end{array}$ & $\begin{array}{l}\text { Daily cost incurred by replacing } \\
\text { the locomotive. }\end{array}$ & $€ /$ day & - & 4000 \\
\hline \multirow{8}{*}{ 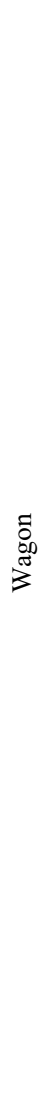 } & $\begin{array}{l}\text { Car/wagon } \\
\text { capacity }\end{array}$ & $\begin{array}{l}\text { Number of } 20 \text { ' containers } \\
\text { (TEU) per car/wagon. }\end{array}$ & $\begin{array}{c}\text { TEU/ } \\
\text { wagon }\end{array}$ & $\begin{array}{c}40^{\prime}=2 \\
60^{\prime}=3 \\
80^{\prime}=4 \\
90^{\prime}=4.5\end{array}$ & - \\
\hline & $\begin{array}{l}\text { Car/wagon } \\
\text { tare weight }\end{array}$ & $\begin{array}{l}\text { Average weight of an empty } \\
\text { car/wagon. }\end{array}$ & Tons & $\begin{array}{c}40^{\prime}=12 \\
60^{\prime}=20.3 \\
80^{\prime}=27.5 \\
90^{\prime}=30\end{array}$ & - \\
\hline & $\begin{array}{l}\text { Car/wagon } \\
\text { max load }\end{array}$ & $\begin{array}{l}\text { Maximum load per one } \\
\text { car/wagon. }\end{array}$ & Tons & $\begin{array}{c}40^{\prime}=33 \\
60^{\prime}=69.7 \\
80^{\prime}=107.5 \\
90^{\prime}=105\end{array}$ & - \\
\hline & $\begin{array}{l}\text { Car/wagon } \\
\text { length over } \\
\text { buffers }\end{array}$ & $\begin{array}{l}\text { Length of the car/wagon from } \\
\text { buffer to buffer. }\end{array}$ & Meters & $\begin{array}{c}40^{\prime}=12 \\
60^{\prime}=20.3 \\
80^{\prime}=27.5 \\
90^{\prime}=30\end{array}$ & - \\
\hline & $\begin{array}{l}\text { Car/wagon } \\
\text { acquisition } \\
\text { value }\end{array}$ & $\begin{array}{l}\text { Price that the buyer will pay to } \\
\text { the car/wagon manufacturer. }\end{array}$ & Euros & - & $\begin{array}{l}40=80,000 \\
60^{\prime}=90,000 \\
80^{\prime}=90,000 \\
90=100,000\end{array}$ \\
\hline & $\begin{array}{l}\text { Car/wagon } \\
\text { useful life }\end{array}$ & $\begin{array}{l}\text { Estimated number of years } \\
\text { car/wagon is likely to remain } \\
\text { in service. }\end{array}$ & Years & - & 40 \\
\hline & $\begin{array}{l}\text { Car/wagon } \\
\text { residual value }\end{array}$ & $\begin{array}{l}\text { Estimated value of the } \\
\text { car/wagon at the end of its } \\
\text { useful life. }\end{array}$ & $\%$ & - & 4 \\
\hline & $\begin{array}{l}\text { Car/wagon } \\
\text { maintenance } \\
\text { costs }\end{array}$ & $\begin{array}{l}\text { Cost covering all car/wagon } \\
\text { maintenance tasks, by year. }\end{array}$ & $\begin{array}{l}€ / \text { wagon } \\
\mathrm{km}\end{array}$ & - & 0.05 \\
\hline
\end{tabular}


Table 1: Continued.

\begin{tabular}{|c|c|c|c|c|c|}
\hline & Variable & Description & Unit & Value & Hypothesis \\
\hline \multirow{10}{*}{ 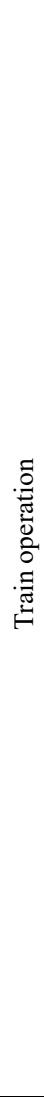 } & $\begin{array}{l}\text { Round trips } \\
\text { per day }\end{array}$ & $\begin{array}{l}\text { Max number round trips/day to } \\
\text { cover the traffic demand in the } \\
\text { selected corridor, taking into } \\
\text { account distance, transit time. }\end{array}$ & $\begin{array}{l}\text { Roundtrips } \\
\text { per day }\end{array}$ & - & $\begin{array}{l}\text { Realistic }=2 \\
\text { Optimistic }=3\end{array}$ \\
\hline & Train schedule & $\begin{array}{l}\text { Number of days per week in } \\
\text { which trains are running. }\end{array}$ & Days/week & - & 5 \\
\hline & Weeks a year & $\begin{array}{l}\text { Number of weeks/year in which } \\
\text { trains can run. }\end{array}$ & Weeks/year & - & 52 \\
\hline & $\begin{array}{l}\text { Total annual } \\
\text { distance } \\
\text { covered }\end{array}$ & $\begin{array}{l}\text { Number of kms run by the rail } \\
\text { services }=\text { door-to-port railway } \\
\text { distance } \times \text { roundtrips per day } \times \\
\text { train schedule } \times \text { weeks } / \text { year. }\end{array}$ & $\mathrm{km}$ & - & 4 \\
\hline & $\begin{array}{l}\text { Container } \\
\text { transfers } \\
\text { among } \\
\text { maritime } \\
\text { terminals }\end{array}$ & $\begin{array}{l}\text { Number of containers } \\
\text { transferred among maritime } \\
\text { terminals by road. }\end{array}$ & $\%$ & - & $0-0.5 \%$ \\
\hline & $\begin{array}{l}\text { Container } \\
\text { transfer costs }\end{array}$ & $\begin{array}{l}\text { Movement of containers at port } \\
\text { and at inland terminals }\end{array}$ & $€ / \mathrm{ITU}$ & - & 40 \\
\hline & $\begin{array}{l}\text { Port terminal } \\
\text { handling } \\
\text { charge }\end{array}$ & $\begin{array}{l}\text { Costs associated with } \\
\text { loading/unloading containers } \\
\text { to/from trains at port terminals. }\end{array}$ & $€ /$ ITU & - & 35 \\
\hline & $\begin{array}{l}\text { Handling } \\
\text { charge at } \\
\text { inland } \\
\text { terminals }\end{array}$ & $\begin{array}{l}\text { Costs associated with } \\
\text { loading/unloading of containers } \\
\text { to/from trains at inland } \\
\text { terminals. }\end{array}$ & $€ / \mathrm{ITU}$ & - & 22 \\
\hline & Train drivers & $\begin{array}{l}\text { Number of train drivers } / \mathrm{d}= \\
\text { Roundtrips per day } \times 2 \text {. }\end{array}$ & $\begin{array}{c}\text { Train } \\
\text { drivers }\end{array}$ & - & 4 \\
\hline & $\begin{array}{l}\text { Annual train } \\
\text { driver cost }\end{array}$ & Cost of train drivers/year. & Euros & - & 80,000 \\
\hline \multirow{4}{*}{ 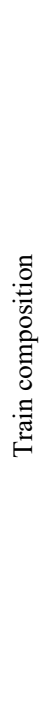 } & $\begin{array}{l}\text { Composition } \\
\text { number }\end{array}$ & $\begin{array}{l}\text { Composition: type and number } \\
\text { of cars/wagons required to cover } \\
\text { the roundtrips/day. }\end{array}$ & $\begin{array}{l}\text { Composi- } \\
\text { tions }\end{array}$ & - & $\begin{array}{c}\text { Realistic }=2 \\
\text { Optimistic }=3\end{array}$ \\
\hline & $\begin{array}{l}\text { Car/Wagon } \\
\text { composition }\end{array}$ & $\begin{array}{l}\text { Number of } 40^{\prime}, 60^{\prime}, 80^{\prime} \text { or } 90^{\prime} \\
\text { cars/wagons. }\end{array}$ & Cars & $\begin{array}{c}* \text { See } \\
\text { Section } 2.2\end{array}$ & - \\
\hline & $\begin{array}{l}\text { Composition } \\
\text { length }\end{array}$ & $\begin{array}{l}\text { Total length of the number and } \\
\text { type of cars/wagons } \\
\text { Composition length }=40^{\prime} \text { car } \\
\text { length } \times 40^{\prime} \text { car number }+60 \text { ' } \\
\text { car length } \times 60^{\prime} \text { car number }+ \\
80 \text { ' car length } \times 80 \text { ' car } \\
\text { number }+90 \text { ' car length } \times 90 \text { ' } \\
\text { car number. }\end{array}$ & Meters & $\begin{array}{c}* \text { See } \\
\text { Section } 2.2\end{array}$ & - \\
\hline & $\begin{array}{l}\text { Composition } \\
\text { tare weight }\end{array}$ & $\begin{array}{l}\text { Weight of each composition } \\
\text { with empty cars/wagons. } \\
\text { Composition tare weight }=40^{\prime}, \\
\text { car tare } \times 40^{\prime} \text { car number }+60^{\prime}, \\
\text { car tare } \times 60^{\prime} \text { car number }+80^{\prime} \\
\text { car tare } \times 80^{\prime} \text { car number }+90^{\prime} \\
\text { car tare } \times 90^{\prime} \text { car number. }\end{array}$ & Tons & $\begin{array}{c}* \text { See } \\
\text { Section } 2.2\end{array}$ & - \\
\hline
\end{tabular}


Table 1: Continued.

\begin{tabular}{|c|c|c|c|c|c|}
\hline & Variable & Description & Unit & Value & Hypothesis \\
\hline \multirow{6}{*}{ 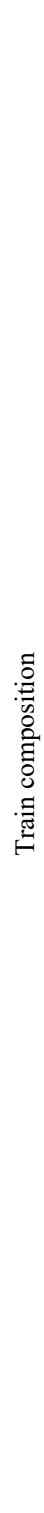 } & $\begin{array}{l}\text { Composition } \\
\text { maximum load }\end{array}$ & $\begin{array}{l}\text { Total maximum load according } \\
\text { to the number and type of } \\
\text { cars/wagons. Composition max. } \\
\text { load }=40 \text { ' car max. load } \times 40^{\prime} \\
\text { car number }+60^{\prime} \text { car max. load } \\
\times 60^{\prime} \text { car number }+80^{\prime} \text { car max. } \\
\text { load } \times 80^{\prime} \text { car max. load }+90^{\prime} \\
\text { car length } \times 90^{\prime} \text { car number. }\end{array}$ & Tons & $\begin{array}{c}* \text { See } \\
\text { Section } 2.2\end{array}$ & - \\
\hline & $\begin{array}{l}\text { Composition } \\
\text { TEU capacity }\end{array}$ & $\begin{array}{l}\text { Composition TEU capacity }= \\
40^{\prime} \text { car capacity } \times 40^{\prime} \text { car } \\
\text { number }+60^{\prime} \text { car capacity } \times 60^{\prime} \\
\text { car number }+80^{\prime} \text { car capacity } \times \\
80^{\prime} \text { car number }+90^{\prime} \text { car } \\
\text { capacity } \times 90^{\prime} \text { car number. }\end{array}$ & TEU & $\begin{array}{c}* \text { See } \\
\text { Section } 2.2\end{array}$ & - \\
\hline & $\begin{array}{l}\text { Composition } \\
\text { ITU capacity }\end{array}$ & $\begin{array}{l}\text { Composition ITU capacity }= \\
\text { Composition TEU capacity } \times \\
\text { container composition. } \\
\text { Composition ITU capacity }= \\
\text { Composition TEU capacity } \times \\
20 \text { ' containers }+ \text { Composition } \\
\text { TEU capacity } \times 40 \text { ' containers } / 2 \\
+ \text { Composition TEU capacity } \times \\
45^{\prime} \text { containers } / 2.5 \text {. }\end{array}$ & ITU & $\begin{array}{c}* \text { See } \\
\text { Section } 2.2\end{array}$ & - \\
\hline & $\begin{array}{l}\text { Composition } \\
\text { estimated } \\
\text { capacity } \\
(100 \% \\
\text { occupancy) }\end{array}$ & $\begin{array}{l}\text { Composition estimated capacity } \\
=\text { composition tare weight }+ \\
\text { (composition ITU capacity } \times \\
\text { full containers } x \text { Full ITU } \\
\text { weight }+(\text { composition ITU } \\
\text { capacity } \times(1 \text { - full containers }) \times \\
\text { ITU tare weight). }\end{array}$ & Tons & $\begin{array}{c}* \text { See } \\
\text { Section } 2.2\end{array}$ & - \\
\hline & $\begin{array}{l}\text { Composition } \\
\text { acquisition } \\
\text { value }\end{array}$ & $\begin{array}{l}\text { Composition acquisition value }= \\
40^{\prime} \text { car acquisition value } \times 40^{\prime} \\
\text { car number }+60^{\prime} \text { car acquisition } \\
\text { value } \times 60^{\prime} \text { car number }+80^{\prime} \\
\text { car acquisition value } \times 80^{\prime} \text { car } \\
\text { number }+90^{\prime} \text { car acquisition } \\
\text { value } \times 90^{\prime} \text { car number. }\end{array}$ & $\begin{array}{l}€ / \text { composi- } \\
\text { tion }\end{array}$ & $\begin{array}{c}* \text { See } \\
\text { Section } 2.2\end{array}$ & - \\
\hline & $\begin{array}{l}\text { Composition } \\
\text { depreciation }\end{array}$ & $\begin{array}{l}\text { Annual composition } \\
\text { depreciation }=(\text { Composition } \\
\text { acquisition value }- \\
(\text { Composition acquisition } \\
\text { value } \times \text { Composition residual } \\
\text { value }) / \text { Composition useful life. }\end{array}$ & $\begin{array}{l}€ / \text { composi- } \\
\text { tion }\end{array}$ & $\begin{array}{c}* \text { See } \\
\text { Section } 2.2\end{array}$ & - \\
\hline \multirow{2}{*}{ 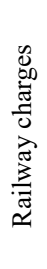 } & $\begin{array}{l}\text { Access to the } \\
\text { railway } \\
\text { infrastructure } \\
\text { charge }\end{array}$ & $\begin{array}{l}\text { Annual charge for using Spanish } \\
\text { railway network, managed by } \\
\text { Adif, the Spanish administrator } \\
\text { of railway infrastructures. }\end{array}$ & $\begin{array}{l}€ / \text { composi- } \\
\text { tion }\end{array}$ & - & 0 \\
\hline & $\begin{array}{l}\text { Railway } \\
\text { capacity } \\
\text { reservation } \\
\text { charge }\end{array}$ & $\begin{array}{l}\text { Charge for reserving the rail } \\
\text { section }(\mathrm{km}) \text { where the train will } \\
\text { run. }\end{array}$ & $\begin{array}{l}€ / \text { composi- } \\
\text { tion } \mathrm{km}\end{array}$ & 0.0724 & - \\
\hline
\end{tabular}


Table 1: Continued.

\begin{tabular}{|c|c|c|c|c|c|}
\hline \multicolumn{2}{|r|}{ Variable } & Description & Unit & Value & Hypothesis \\
\hline \multirow{2}{*}{ 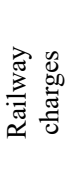 } & $\begin{array}{l}\text { Rail traffic } \\
\text { charge }\end{array}$ & $\begin{array}{l}\text { Charge for the real use of the } \\
\text { capacity reserved. }\end{array}$ & $\begin{array}{l}€ / \text { composi- } \\
\text { tion } \mathrm{km}\end{array}$ & 0.1032 & - \\
\hline & $\begin{array}{l}\text { ACA services } \\
\text { charge }\end{array}$ & $\begin{array}{l}\text { Charge for the Additional, } \\
\text { Complementary and Auxiliary } \\
\text { services provided by Adif. }\end{array}$ & $\begin{array}{c}\text { Euros/round } \\
\text { trip }\end{array}$ & - & 400 \\
\hline \multirow{4}{*}{ 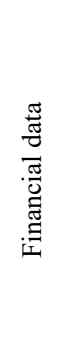 } & Roi & $\begin{array}{l}\text { Roi measures the gain or loss } \\
\text { generated on the investment, } \\
\text { related to the amount of money } \\
\text { invested in the locomotive and } \\
\text { cars/wagons. }\end{array}$ & $\%$ & - & 2.5 \\
\hline & Financing & Funds for business activities. & $\%$ & - & 0 \\
\hline & $\begin{array}{l}\text { Financing } \\
\text { period }\end{array}$ & $\begin{array}{l}\text { Time over which the rail } \\
\text { company borrows money. }\end{array}$ & Years & - & 10 \\
\hline & $\begin{array}{l}\text { Annual } \\
\text { interest rate }\end{array}$ & $\begin{array}{l}\text { Interest rate paid on the } \\
\text { investment. }\end{array}$ & $\%$ & - & 3.5 \\
\hline
\end{tabular}

ITU: intermodal transportation unit; km: kilometers; roi: return on investment; 1 TEU: a 20' container.

Seven scenarios were defined, based on the variables and hypotheses listed in Table 1. The differences among the scenarios selected are in terms of the following variables, as described in Table 1:

- Traffic-container type: Container composition; Full containers; Full ITU weight; ITU tare weight; Full TEU weight.

- Locomotive: Locomotive type; Maximum towable load; Locomotive acquisition value; Locomotive annual depreciation.

- Train operation: Roundtrips per day; Total annual distance covered; Train drivers.

- Train composition: Composition number.

\subsection{Optimal composition model}

The optimal number and type of train cars/wagons was obtained in two steps, maximizing first the capacity and minimizing next the total tare weight of the train composition. The optimization problem was solved using the Microsoft Excel Solver.

- First step:

$$
\operatorname{Max} C=\sum_{\forall i} n_{i} \cdot c_{i}
$$

Subject to:

$$
\sum_{i} n_{i} \cdot k_{i}+\sum_{i} n_{i} \cdot c_{i}(\varphi \cdot \sigma+(1-\varphi) \cdot \omega) \leq T \text { (max towable load limit). }
$$

(ii) $\quad \alpha \sum_{i} n_{i} \cdot c_{i} \geq n_{60}$, (limit of $60 \mathrm{ft}$ wagons, in order to avoid empty spaces).

(iii) $\quad \sum_{i} n_{i} \cdot l_{i} \leq L$ (max length limit).

Parameters and variables:

- Train composition

$$
\begin{aligned}
& i=\text { type of car/wagon } \quad i \in\left\{40^{\prime} ; 60^{\prime} ; 80^{\prime} ; 90^{\prime}\right\} \\
& n_{i}=\text { number of cars/wagons of type } i \text { (decision variables) }
\end{aligned}
$$


$c_{i}=$ capacity in number of TEU of one car/ wagon of type $i$

$k_{i}=$ tare weight of one car/wagon of type $i$

$l_{i}=$ length of one car/wagon of type $i$

$T=$ Maximum towable load (depends on the locomotive and the maximum slope in the route)

$L=$ Maximum length of the train composition allowed on the route

$C=$ Train capacity in number of TEU

- $\quad$ Traffic composition

$\varphi=$ percentage of full TEU

$\alpha=$ percentage of $20-$ foot containers

$\sigma=$ average weight per TEU for full containers

$\omega=$ average tare weight per TEU for empty containers

- Second step:

$$
\operatorname{Min} \sum_{\forall i} n_{i} \cdot k_{i}
$$

Subject to:

$$
\sum_{i} n_{i} \cdot c_{i} \geq C \text { (min capacity limit) }
$$

The optimal solution (wagon composition) for all the defined scenarios is to consider the $80 \mathrm{ft}$ of wagons, allowing to load $4 \times 20 \mathrm{ft}$ containers, $2 \times 40 \mathrm{ft}$, or a combination of a $40 \mathrm{ft}$ unit in the middle and $2 \times 20 \mathrm{ft}$ units at the beginning and end platform.

\section{COST ANALYSIS}

Once the optimal composition of the number and type of train cars/wagons had been obtained for each scenario, a cost analysis was carried out from the point of view of the railway undertaking that operates the JIT rail shuttle service between the port of Valencia and Zaragoza (Table 2). This annual cost analysis for each scenario will allow us to select the option that minimizes the cost per unit of TEU transported, which is to be charged to the final customer.

\begin{tabular}{|c|c|c|c|}
\hline \multicolumn{2}{|r|}{ Cost } & Description & Unit \\
\hline \multirow{3}{*}{ 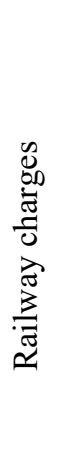 } & $\begin{array}{l}\text { Access to } \\
\text { railway } \\
\text { infrastructure } \\
\text { annual charge }\end{array}$ & $\begin{array}{l}\text { Annual charge for the use of the Spanish } \\
\text { railway network managed by Adif, the } \\
\text { Spanish administrator of the railway } \\
\text { infrastructures. }\end{array}$ & $\begin{array}{l}€ / \text { composi- } \\
\text { tion-year }\end{array}$ \\
\hline & $\begin{array}{l}\text { Railway } \\
\text { capacity } \\
\text { reservation, } \\
\text { annual charge }\end{array}$ & $\begin{array}{l}\text { Railway capacity reservation annual charge } \times \\
\text { total annual distance covered. }\end{array}$ & $\begin{array}{l}€ / \text { composi- } \\
\text { tion-year }\end{array}$ \\
\hline & $\begin{array}{l}\text { Rail traffic, } \\
\text { annual charge }\end{array}$ & $\begin{array}{l}\text { Rail traffic charge } \times \text { total annual distance } \\
\text { covered. }\end{array}$ & $\begin{array}{l}€ / \text { composi- } \\
\text { tion-year }\end{array}$ \\
\hline
\end{tabular}

Table 2: Cost description. 
Table 2: Continued.

\begin{tabular}{|c|c|c|c|}
\hline \multicolumn{2}{|r|}{ Cost } & Description & Unit \\
\hline \multirow{4}{*}{ 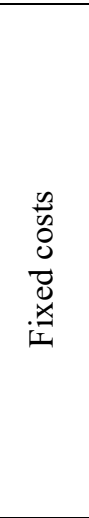 } & $\begin{array}{l}\text { Locomotive } \\
\text { annual } \\
\text { depreciation }\end{array}$ & $\begin{array}{l}\text { Locomotive annual depreciation }= \\
\text { (locomotive acquisition value }- \text { (locomotive } \\
\text { acquisition value } \times \text { locomotive residual } \\
\text { value) } / \text { locomotive useful life. }\end{array}$ & $\begin{array}{c}€ / \text { composi- } \\
\text { tion-year }\end{array}$ \\
\hline & $\begin{array}{l}\text { Replacement } \\
\text { locomotive } \\
\text { annual cost }\end{array}$ & $\begin{array}{l}\text { Replacement locomotive annual cost }=\text { days } \\
\text { of a replacement locomotive } \times \text { replacement } \\
\text { locomotive daily cost. }\end{array}$ & $\begin{array}{l}€ / \text { composi- } \\
\text { tion-year }\end{array}$ \\
\hline & $\begin{array}{l}\text { Composition } \\
\text { acquisition } \\
\text { value }\end{array}$ & $\begin{array}{l}\text { Rail car/wagon acquisition value } \times \text { car/wagon } \\
\text { composition. }\end{array}$ & $\begin{array}{l}€ / \text { composi- } \\
\text { tion-year }\end{array}$ \\
\hline & $\begin{array}{l}\text { Train driver } \\
\text { annual cost }\end{array}$ & Train drivers $\times$ annual train driver cost. & $\begin{array}{c}\text { €/composi- } \\
\text { tion-year }\end{array}$ \\
\hline \multirow{3}{*}{$\begin{array}{l}\frac{n}{0} \\
0 \\
0 \\
0 \\
\frac{0}{0} \\
\cdot \frac{\pi}{\Xi} \\
>\end{array}$} & $\begin{array}{l}\text { Fuel } \\
\text { consumption } \\
\text { annual cost }\end{array}$ & $\begin{array}{l}\text { Fuel consumption } \times \text { Fuel price } \times \text { total annual } \\
\text { distance covered. }\end{array}$ & $\begin{array}{l}€ / \text { composi- } \\
\text { tion-year }\end{array}$ \\
\hline & $\begin{array}{l}\text { Locomotive } \\
\text { maintenance } \\
\text { annual cost } \\
\end{array}$ & $\begin{array}{l}\text { Locomotive maintenance cost per } \mathrm{km} \times \text { total } \\
\text { annual distance covered. }\end{array}$ & $\begin{array}{l}€ / \text { composi- } \\
\text { tion-year }\end{array}$ \\
\hline & $\begin{array}{l}\text { Car/Wagon } \\
\text { maintenance } \\
\text { annual cost }\end{array}$ & $\begin{array}{l}\text { Car/wagon annual maintenance cost } \times \\
\text { car/wagon composition } \times \text { total annual distance } \\
\text { covered. }\end{array}$ & $\begin{array}{l}€ / \text { composi- } \\
\text { tion-year }\end{array}$ \\
\hline \multirow{4}{*}{ 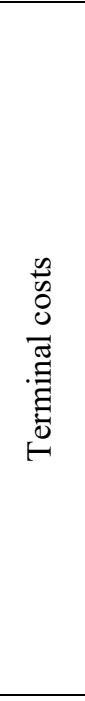 } & $\begin{array}{l}\text { Terminal } \\
\text { handling } \\
\text { annual } \\
\text { charge at } \\
\text { port } \\
\text { terminals }\end{array}$ & $\begin{array}{l}\text { Terminal handling charge at port terminals } \times \\
\text { composition ITU capacity. }\end{array}$ & $\begin{array}{l}€ / \text { composi- } \\
\text { tion-year }\end{array}$ \\
\hline & $\begin{array}{l}\text { Handling } \\
\text { annual } \\
\text { charge at } \\
\text { inland } \\
\text { terminals }\end{array}$ & $\begin{array}{l}\text { Handling charge at inland terminals } \mathrm{x} \\
\text { Composition ITU capacity. }\end{array}$ & $\begin{array}{l}€ / \text { composi- } \\
\text { tion-year }\end{array}$ \\
\hline & $\begin{array}{l}\text { Annual } \\
\text { ACA } \\
\text { services } \\
\end{array}$ & ACA services $\times$ roundtrips/year. & $\begin{array}{l}€ / \text { composi- } \\
\text { tion-year }\end{array}$ \\
\hline & $\begin{array}{l}\text { Container } \\
\text { transfer } \\
\text { annual costs } \\
\end{array}$ & $\begin{array}{l}\text { Container transfers among maritime terminals } \\
\times \text { composition ITU capacity } \times \text { container } \\
\text { transfer costs. }\end{array}$ & $\begin{array}{l}€ / \text { composi- } \\
\text { tion-year }\end{array}$ \\
\hline 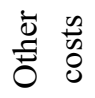 & $\begin{array}{l}\text { Other annual } \\
\text { costs }\end{array}$ & $\begin{array}{l}\text { Management, maintenance, etc. } \\
15 \% \text { of the total of the previous costs. }\end{array}$ & $\begin{array}{l}€ / \text { composi- } \\
\text { tion-year }\end{array}$ \\
\hline
\end{tabular}


Given that this JIT rail shuttle service will be the first operating in Spain, the more realistic option in the short term would be the scenario of two roundtrips/day, and the following main characteristics:

- Locomotive type: EURODUAL; Maximum towable load: 1,173 tones.

- Roundtrips per week: 10 (2 daily roundtrips).

- Composition number: 2.

- Car/wagon composition: 40' car $/$ wagon $=0 ; 60$ ' car $/$ wagon $=0 ; 80^{\prime} \mathrm{car} /$ wagon $=18$; 90 ' car/wagon $=0$.

- Composition length: 475.

- Occupancy composition: $100 \%$.

- Cost per TEU transported: 95.37 Euros/TEU.

\subsection{Sensitivity analysis}

A sensitivity analysis has been carried out on the cost per TEU transported ( $€ /$ TEU), applying stochastic simulation to the input variables: an average was established for each parameter (the value that displays the highest expected frequency) along with a confidence interval (CI) using the minimum and maximum expected values for this factor. Once the average, minimum and maximum values were attained, the probability distribution of expected changes in the parameter was studied, and the stochastic variability interval or margin for error was established, with different levels of clearly defined probability.

As demonstrated in Table 3, the most determinant factor in the cost per TEU transported is the Terminal Handling Charge (THC) at port terminals, with a positive sign of $18.2 \%$. The main objective of the JIT rail shuttle service is to reduce handling movements at container terminals, resulting in both cost and time savings.

The next most determinant variable is composition occupancy, with a negative sign. The greater the number of containers transported per train, the lower the expected cost per TEU.

Table 3: Sensitivity analysis, Euros/TEU.

\begin{tabular}{|l|c|}
\hline \multicolumn{1}{|c|}{ Variable } & $\begin{array}{c}\text { Percentage of variance in } \\
€ / \text { TEU }\end{array}$ \\
\hline Port terminal handling charge & $18.2 \%$ \\
\hline Occupancy composition (\%) & $-17.6 \%$ \\
\hline Fuel price & $11.8 \%$ \\
\hline ACA services charge & $11.7 \%$ \\
\hline Inland terminal handling charge & $8.2 \%$ \\
\hline Daily round trips & $-5.6 \%$ \\
\hline Composition number & $-5.6 \%$ \\
\hline Transfers among terminals & $4.7 \%$ \\
\hline Train driver cost, annual & $3.5 \%$ \\
\hline Locomotive's useful life & $-3.0 \%$ \\
\hline Other costs & $2.3 \%$ \\
\hline Container transfer costs & $2.1 \%$ \\
\hline
\end{tabular}




\section{INFORMATION SYSTEM REQUIREMENTS}

This section identifies the information requirements required for the implementation of a JIT rail shuttle service. The proposed JIT rail shuttle service will solve existing problems affecting rail freight services at ports:

1. Lack of information in the unloading list, related to the containers to be loaded into train services.

2. Delays in the container information to be loaded onto trains: port terminals receive information about whether the container will be loaded onto a rail service (in unloading list) once the vessel arrives; and then the container is unloaded and moved to storage areas.

3. Railway operators do not share slots in the different trains. Therefore, the available resources (assets) are not fully used.

\subsection{Current situation}

At present, the different agents participating in rail transport procedures use the Port Community Systems (PCS) as the open and neutral electronic platform that allows for a safe and smart information exchange between the public and private agents. The main documents exchanged are transport orders (transport instructions), acceptance/release orders, the Train Loading/Discharge List and the train composition; however, although container terminals do receive information on what to load onto a train by electronic means, this information is received after the containers are discharged from the vessel. Therefore, imported containers must wait in the container yard before they are transported to the train loading area, leading to unnecessary movements and to idle time prior to final delivery.

\subsection{New information requirements}

The new JIT Rail Shuttle Service requires additional changes that affect the current information flowing between different agents. These changes will reduce the number of handling movements carried out in the terminal, seeking maximum optimization through a "Just-In-Time" service. The latter involves the provision of certain information in advance to the port container terminals; so that just as the containers are being discharged from the vessel, they can be positioned in the rail terminal loading areas, without the need for storage in the container yard prior to loading.

The proposed procedure is: before the arrival of the vessel, shipping agencies will indicate in the Discharge List, which containers should be loaded into the shuttle service, given their priority. With this information, port container terminals will generate Shuttle Loading Lists that will be transmitted to the railway companies through the PCS. At this time, the railway companies will validate the lists, so that the port terminal may begin its handling operations. Once the handling operations finish, port terminals will send a Loading List confirmation to all the different agents who are involved in the logistics chain (shipping agencies, railway operators, freight forwarders, and such).

\section{BUSINESS MODEL}

Business models determine the value proposition of a given product or service, how it will be exploited, who will be the main customers and partners, and which will be the main sources of revenue and marketing channels. In the case of the JIT Rail Shuttle Service, it is a collaborative business model whose main value proposal consists of minimizing idle time at 
container terminals, achieving a ship-to-train interconnection, without additional movements occurring in the container terminal yard.

In this regard, a "SHUTTLE" authority will be created to manage the capacity (number of trains) that is added to the service, and the "slots" or spaces in the train that are sold to different railway operators. The shuttle authority is to be a private, public or PPP company that owns locomotives and train cars/wagons. This company issues tender contracts to select railway companies that want to operate the shuttle service at a certain price, while maintaining the established quality of service. Once selected, railway companies are paid in advance by the SHUTTLE authority for those services that will be carried out during an established period of time. In addition, after a railway company is selected, the SHUTTLE authority issues a bidding process, to sell the available slots among the interested railway operators/freight forwarders.

Rail operators/freight forwarders make their bids to be able to trade a certain percentage of the available slots in the shuttle. Afterwards, the SHUTTLE authority studies the offers and chooses the best bids (those that guarantee a lowest $€ /$ TEU for the shippers).

On the other hand, a financial compensation system is managed by the SHUTTLE entity, so that railway operators can use more/less capacity than the one assigned in the bidding process. In this regard, the new business model includes a mechanism for slot exchange between the different railway operators, so that if one of them does not fill all of its available capacity on a given train, this can be used by another operator, and vice versa. In this way, the trains are more likely to be filled, and there is greater flexibility and lower financial risk for railway operators.

At the end of each month, depending on the real occupation used, the Port Community System extracts a series of reports, with which the SHUTTLE entity can make corresponding adjustments. Under this model, railway operators achieve greater flexibility, frequency and regularity of service; and railway companies achieve greater train utilization by better amortizing the available resources/assets.

It is important to point out that the proposed business model requires an important technological leap through the intelligent compensation module, and through the new processes and information flows that have already been described in Section 3.2. These innovations make it easier for operations to be planned, even before the arrival of the ship at port, speeding up the transfer of containers from the ship to the train and destination, while minimizing the dwell time in port.

Finally, the proposed model is based on the basic principles of the physical internet, through which all participants in the supply chain act as an interconnected network through which information is transferred and shared, maximizing the efficiency of operations and achieving the just-in-time services; as well as the elimination of those superfluous costs generated by current or previously inefficient processes.

\section{CONCLUSIONS}

The Port Authority of Valencia aims to increase the rail modal share for import/export cargo, through innovative solutions that can help to foster the rail transport mode.

One of the solutions proposed is the direct unloading of containers from the vessel onto trains, minimizing handling movement and operating as an "air bridge" does at airports, so that the shuttle does its round trips within a day and in which the containers are loaded onto the first available "Just-In-Time" rail shuttle service. The key successful factor for this JIT Rail Shuttle implementation will be the cost, which will help attract shippers to use rail instead of the road transportation mode. Thus, the optimal solution will be the one that minimizes the cost per unit transported (minimum cost per TEU). 
Firstly, in order to get the optimum physical and operational solution, a set of hypotheses was analyzed. These hypotheses resulted in seven scenarios for which, firstly, obtaining the optimal composition of the shuttle train based on the number and type of train cars/wagons was needed. The optimal solution found for the seven defined scenarios was: $80 \mathrm{ft}$ of train cars/wagons, which allows loading $4 \times 20 \mathrm{ft}$ of containers, or a combination of a $40 \mathrm{ft}$ unit in the middle and $2 \times 20 \mathrm{ft}$ units at the beginning and end of the platform.

Secondly, a cost analysis was carried out from the point of view of the railway undertaking, which operates the JIT rail shuttle service in the selected corridor. Given the fact that this JIT rail shuttle service will be the first rail shuttle operating in Spain, the scenario selected establishes two round trips per day as a realistic option within the short term. Besides this, we performed a sensitivity analysis on the cost per TEU transported ( $€ / T E U)$, applying stochastic simulation to see which are the most relevant parameters that affect it. In this sense, we determined that the port and inland terminal handling charges, the train occupancy ratio and the fuel price can explain almost $50 \%$ of the cost per unit transported.

Another important issue for the implementation of the shuttle service is the information exchange involved between the actors (shipping agents, port-rail-inland terminals/container terminals, freight forwarders, railway operators and other railway undertakers). The implementation of the new JIT Rail Shuttle Service requires important changes that would affect the current information flow. The port terminals will assume a new role in the loading/unloading procedure, since they are the ones who will be managing which containers are transported by the shuttle. For this purpose, the Valenciaport Port Community System and the port Terminal Operating System (TOS) will also play key roles.

Finally, the business model proposed for the operation of the JIT Rail Shuttle Service is a collaborative model between the different actors; in which a "SHUTTLE" entity will manage both the capacity of trains that is added to the service, and the "slots" (spaces in the train) that are sold to the different railway operators.

Both the railway operators and railway undertakings will receive benefits from this model: the railway operators will have the possibility to exchange slots, achieving greater flexibility, frequency and regularity of their service; and the railway undertakings will increase train occupancy, minimizing the cost per unit transported.

\section{ACKNOWLEDGEMENT}

The authors are grateful for the funding received from the H2020 Programme through the COREALIS Project (Capacity with a positive environmental and societal footprint: ports in the future era).

\section{REFERENCES}

[1] Acciaro, M. \& McKinnon, A., Efficient hinterland transport infrastructure and services for large container ports. ITF/OECD Discussion Paper 19. Presented at RT Port Investment and Container Shipping Markets Roundtable, Santiago, Chile, 2013.

[2] de Langen, P.W., Ensuring hinterland access. The role of port authorities. ITF/OECD Discussion Paper 11. Presented at Research Round Table: "Seaport Competition and Hinterland Connections”, Paris, France, 2008.

[3] European Commission, White Paper on transport, Roadmap to a single European transport area - towards a competitive and resource-efficient transport system. 2011.

[4] Mountreuil, B., Toward a physical internet: meeting the global logistics sustainability grand challenge. Logistic Research, 3(2-3), pp. 71-87, 2011. 